\title{
COEXISTENCE OF CONDUCTING AND NONCONDUCTING PHASES ON THE METALLIC SIDE OF THE MOTT TRANSITION IN PHOTOINJECTED SEMICONDUCTORS
}

\author{
A. Sergio Esperidião, $\dagger$ Aurea R. Vasconcellos $\ddagger$ and Roberto Luzzi $\ddagger$ \\ †Departamento de Física Geral, Universidade Federal da Bahia, 40211-Salvador, BA, Brazil \\ †Departamento de Física do Estado Sólido e Ciência de Materiais, Instituto de Física; \\ Universidade Estadual de Campinas (UNICAMP), C.P. 6165; 13081-Campinas, SP, Brazil
}

(Received 4 September 1991; accepted 29 January 1992)

\begin{abstract}
The nonequilibrium steady state of a direct gap semiconductor is studied under high levels of photoexcitation by continuous laser light. The stability of the uniform steady state of itinerant carriers is probed resorting to linear normal mode analysis of the nonlinear equations of evolution for the carrier charge density. Such analysis leads to the determination of the wavevector dependent electronic contribution to the dielectric function. Examination of its behavior allows us to show that in the extremely degenerate regime the carrier system becomes nonmetallic, and displays a coexistence of metallic and nonmetallic phases on leaving that regime: itinerant carriers move in the background of an extended state of bounded electron and hole charge densities. This introduces a new view of Mott transition in photoinjected semiconductors. This complex behavior is a result of collective together with dissipative effects in the far from equilibrium carrier system governed by nonlinear dynamic laws.
\end{abstract}

Keywords: Mott transition, nonequilibrium semiconductors, nonequilibrium thermodynamics, metallic to nonmetallic transition.

\section{INTRODUCTION}

In the last decades it has been shown that open systems governed by nonlinear dynamical laws, when sufficiently far from equilibrium, may display unexpected complex behavior $[1,2]$. In far-fromequilibrium conditions a system in a steady state plays an important role. Whereas isolated systems in equilibrium are characterized by a maximum of the thermodynamic entropy, steady states in open systems near equilibrium (Onsager's linear regime) are characterized by a minimum production of entropy (Prigogine's theorem) [3]. On the other hand, when far away from equilibrium (nonlinear regime) open systems are susceptible to present, beyond a critical point of excitation, self-organized ordered patterns in the form of temporal or spatial organizations (e.g. chemical clocks, chemical waves, morphological transitions, etc.), or to attain a new uniform steady state (e.g. negative differential resistivity, lasers, etc.). This is a remarkable aspect of nonlinearity in nonequilibrium thermodynamics, called dissipative structures after Prigogine $[1,4]$.

The considerations just stated suggest that semiconductors under high levels of excitation, whose nonequilibrium thermodynamic state is governedin this strongly dissipative regime-by nonlinear evolution laws, requirc an analysis in order to indicate the possibility of some kind of complex behavior when driven far removed from equilibrium. We consider a semiconductor sample illuminated by strong continuous laser light, with a photon energy higher than the band gap. Electron-hole pairs are produced, and we assume that the laser intensity is strong enough to produce concentrations on the metallic side of the Mott transition (typically $n \gtrsim 10^{16} \mathrm{~cm}^{-3}$ ). A double plasma is then formed, i.e. itinerant electrons and holes (carriers) moving in the lattice background. After a certain transient period the carriers form a uniform steady state, characterized by photoconcentration $n$ and a carrier quasitemperature $T^{*}$ [5].

We look for the possibility of the instability of such a uniform steady stage against the formation of a Prigogine-like dissipative structure in the charge density, either nonuniform (a morphological transition) or another different uniform steady state (e.g. bistability or other kind). For that purpose we look for the equations of evolution for the local carrier charge density; in fact we consider its Fourier amplitudes which allow easier handling of such equations in the second quantization representation. We resort to the use of a nonlinear quantum transport theory based on the nonequilibrium 
statistical operator method (NSOM). This technique, described with some detail in [6], has had extensive applications, being previously used by our group in studies of ultrafast relaxation [7] and transport processes [8] in HEPS, where it has been briefly reviewed (see also [5]). We resort to Zubarev's approach [9] and the so-called linear theory of relaxation [10].

We derive, using the NSOM and RPA, the nonlinear equations of evolution for the Fourier amplitudes of the carrier charge density, the nonlinearities consisting in bilinear terms that couple the different Fourier amplitudes. Zero value for them characterizes the uniform state of reference. We perform linear normal mode analysis [11] to find that the uniform steady state is stable against the formation of spatial order in the system. In the process we show that the normal mode analysis is equivalent to looking for the behavior of the wave-vector dependent static dielectric function, $\epsilon(\vec{Q})$. Spatial instability would correspond to, for a certain $\vec{Q}$, that $\epsilon(\vec{Q})$ at a certain distance from equilibrium (a critical laser intensity) vanishes and on increasing the intensity becomes negative; this is not the case.

But, such a calculation of $\epsilon(\vec{Q})$ allows us to show that there exists a condensation of the itinerant carriers in a nonmetallic state in the extremely degenerate regime. The static dielectric function $\epsilon(\vec{Q}=0)$, which is infinite for a system of itinerant electrons, thus characterizing the metallic state, becomes finite, i.e. characteristic of a dielectric state. On leaving the highly degenerate regime, it recovers its infinite value, but for non-null $\vec{Q}$ remains smaller than the value of the Lindhart (RPA) dielectric function for the metallic system of free electrons and holes. This suggests the coexistence of itinerant and condensed carriers in the system, i.e. coexistence of metallic and a nonmetallic nonequilibrium thermodynamic phases. Using a simple model of two fluids, we estimate the concentration of carriers in the condensed dielectric phase: this number decreases very rapidly (exponentially) with a very low characteristic temperature, for each concentration, at least in our numerical calculation appropriate for GaAs.

In the mixed regime, itinerant carriers move in the background of an extended state of bounded electron and hole charge densities in a dielectric phase. The condensed polarizable state seems to correspond to the condensation of the electrons and holes in two interpenetrated, and clamped together, infinite wavelength lattices, presumably forming extended Frenkel-like excitons.

In the next section we show the calculations and numerical results for the case of a GaAs sample. In the final section we summarize and further discuss the results.

\section{STABILITY ANALYSIS}

Consider an intrinsic direct-gap polar semiconductor sample illuminated by a continuous laser beam, with power flux $I_{\mathrm{L}}$ and photon frequency $\omega_{\mathrm{L}}$. The photogenerated electron-hole pairs (carriers) created in photon absorption processes are supposed to be on the metallic side of the Mott transition, i.e. are describable as itinerant carriers. The open system of carriers relax the excess energy of equilibrium received from the laser source to the lattice, while their total number varies as it grows by photon absorption but diminishes in recombination processes. We assume a constant laser intensity throughout the volume of laser light focalization, and stimulated emission and self-absorption are neglected in comparison with spontaneous recombination. The system Hamiltonian consists of the carrier energy operator (clectron and hole band energies in the effective mass approximation plus Coulomb interaction dealt with in the random phase approximation); the Hamiltonian of the photon field; and the energy operators describing the interaction of carriers with lattice vibrations and with the laser and recombination radiation fields. We use an Einstein (dispersionless) model for the optical phonons and a Debye model for the acoustic phonons. Further, the carrier-radiation interaction is treated in the dipole approximation, with the photon field described by a classical field incorporated in the carrier Hamiltonian. The laser source and the thermal bath, the latter at temperature $T_{0}$, are taken as ideal reservoirs, i.e. they are assumed to remain in stationary unaltered condition while constantly coupled with the open semiconductor sample.

We resort to the use of the NSOM [6] in Zubarev's approach [9] to describe the nonequilibrium macroscopic state of this system. As shown in previous publications the system of carriers attains a very rapid internal thermalization (subpicosecond time scale) as a result of Coulomb interaction [12] and can be described in terms of a quasi-temperature and quasi-chemical potentials (or photoconcentration) $[5,7,8]$. On the other hand, the optical phonons receive part of the excess energy pumped by the laser source on the carrier system and are warmed up. This occurs in a differentiated way with modes in a restricted region of Brillouin zone being preferentially excited, but final thermalization of the optical phonon field and carriers follows in the 10-fold picosecond time scale [13]. From then on, the optical phonons can be described by a unique 
quasi-temperature (cqual to that of the carriers). This is followed by a tendency of the carriers and optical phonon subsystems to attain equilibrium with the thermal bath via anharmonic interactions with the acoustic phonons and heat diffusion of the latter to the reservoir. It is expected that in a short time (nanosecond time scale) the quasi-temperatures of the subsystems ncarly equal the reservoir temperature. Therefore, the nonequilibrium photoinjected system is characterized by temperature $T_{0}$ and a uniform concentration $n$ [5]. The latter attains a steady-state value in times of the order of nano- to milliseconds, depending on the intensity of the lase source [5].

Applying the nonlinear quantum transport theory derived from Zubarev's NSOM [9] in the so called linear theory of relaxation (LTR) [10] an equation is obtained [5] that gives $n$ in terms of the laser power flux $I_{\mathrm{L}}$, namely

$$
\begin{aligned}
\frac{2 \pi}{\hbar} \sum_{k, \dot{q}}\left|U^{\mathrm{R}}(\vec{k}, \vec{q})\right|^{2} f_{k}^{\mathrm{e}} f_{k}^{\mathrm{h}} & \delta\left(\epsilon_{\vec{k}}^{X}+E_{\mathrm{G}}-\hbar \omega_{\dot{q}}\right) \\
& =\alpha\left(\omega_{\mathrm{L}}\right) I_{\mathrm{L}}\left(1-f_{\mathrm{L}}^{\mathrm{e}}-f_{\mathrm{L}}^{\mathrm{h}}\right),
\end{aligned}
$$

where $U^{\mathrm{R}}$ is the matrix element for the recombination process; $\epsilon_{k}^{x}=\hbar^{2} k^{2} / 2 m_{x}$, with $m_{x}$ being the excitonic mass $m_{x}^{-1}=m_{\mathrm{e}}^{-1}+m_{\mathrm{h}}^{-1}\left(m_{\mathrm{e}(\mathrm{h})}\right.$ are the effective masses of electrons and of holes); $\omega_{\mathrm{q}}$ is the frequency of the photons produced in recombination processes and given by $c q / \epsilon_{\infty}^{1 / 2}$, with $\epsilon_{\infty}$ the high frequency background dielectric constant; $f_{k}^{\mathrm{e}(\mathrm{h})}$ are Fermi-Dirac distribution functions at temperature $T_{0}$ and quasichemical potentials $\mu_{\mathrm{e}}$ and $\mu_{\mathrm{h}}$ and energies $\epsilon_{k}^{\mathrm{e}(\mathrm{h})}=$ $\hbar^{2} k^{2} / 2 m_{\mathrm{e}(\mathrm{h})} ; f_{\mathrm{L}}^{\mathrm{e}(\mathrm{h})}$ are the Fermi-Dirac distributions for energies $\left(m_{x} / m_{\mathrm{e}(\mathrm{h})}\right)\left(\hbar \omega_{\mathrm{L}}-E_{\mathrm{G}}\right) ; E_{\mathrm{G}}$ is the energy gap; and $\alpha\left(\omega_{\mathrm{L}}\right)$ the photon absorption coefficient at the laser frequency. The concentration $n$ and quasichemical potentials $\mu_{\mathrm{e}(\mathrm{h})}$ are related, in the internally thermalized carrier system, by the well-known expression [14]

$$
n=n_{\mathrm{e}}^{0} F_{1 / 2}\left(\beta \mu_{\mathrm{e}}\right)=n_{\mathrm{h}}^{0} F_{1 / 2}\left(\beta \mu_{\mathrm{h}}\right),
$$

where $F_{1 / 2}$ is the Fermi function of index one half and

$$
n_{\mathrm{e}(\mathrm{h})}^{0}=2\left[2 \pi m_{\mathrm{e}(\mathrm{h})} / \hbar^{2} \beta\right]^{3 / 2}
$$

with $\beta=1 / k T^{*}$.

In this way, at the macroscopic thermodynamic level, the uniform steady state of the system is completely characterized. Next, as noted in the Introduction, we turn our attention to the study of the behavior of the local charge density of the carrier system. For that purpose we enlarge the NSOMbasic set of macrovariables with the inclusion of the non-diagonal elements of the single-particle density matrix, namely

$$
\begin{aligned}
& n_{k \bar{Q}}(t)=\operatorname{Tr}\left\{C_{k+Q}^{\ddagger} C_{k} \rho_{\epsilon}(t)\right\} \\
& n_{k \bar{Q}}^{b}(t)=\operatorname{Tr}\left\{h_{-k-\bar{Q}} h_{k}^{\ddagger} \rho_{\epsilon}(t)\right\}
\end{aligned}
$$

where $C(C \dagger)$ and $h(h \dagger)$ are annihilation (creation) operators for electrons and holes, respectively, in plane-wave states, used to approximate Bloch-band states in the neighbourhood of the zone centre. Further, $\rho_{\epsilon}(t)$ is Zubarev's NSO appropriate for this problem, built according to the rules of the NSOM $[6,9]$ in terms of the NSOM-auxiliary operator $\bar{\rho}(t)$ for this case, namely

$$
\bar{p}(t)=\exp \left\{-S_{0}(t)-\delta S(t)\right\}
$$

Here, $S_{0}$ is the contribution associated to the uniform state (see eqn (14) in [5]), and

$$
\begin{aligned}
& \delta(t)=\delta \phi(t)+\sum_{k, \emptyset}\left[F_{k \underline{Q}}(t) C_{k+\emptyset}^{\ddagger} C_{\vec{k}}\right. \\
& \left.+F_{k \bar{Q}}^{\mathrm{h}}(t) h_{-k-\bar{Q}}^{\mathrm{h}} \mathrm{h}_{-\bar{k}}^{\dagger}\right] \text {, }
\end{aligned}
$$

where $\phi_{0}+\delta \phi$ ensures the normalization of the statistical operator of eqn (5), and $F_{k \delta}^{\mathrm{e}(h)}$ are the associated Lagrange multipliers to the operators of eqns (4) that appear in the variational formulation of the NSOM (6).

The equations of motion for the quantities of eqns (4), to be coupled to eqns (1) and (2), in Zubarev's approach and LTR, and using RPA to deal with the Coulomb interaction between carriers, are

$$
\begin{aligned}
& i \hbar \frac{\partial}{\partial t} n_{k \bar{Q}}^{e}(t)=\Delta E_{k \bar{Q}}^{e} n_{k \bar{Q}}^{e^{\prime}}(t)-2 V(Q) \Delta f_{k \bar{Q}}^{e} n(\vec{Q}, t) \\
& +i B_{k Q}^{\mathrm{ch}} n_{k Q}^{\mathrm{h}}(t)-i B_{k Q}^{\mathrm{e}} n_{k \emptyset}^{\mathrm{e}}(t)+N_{k Q}^{\mathrm{e}}(t),
\end{aligned}
$$

$$
\begin{aligned}
& i \hbar \frac{\partial}{\partial t} n_{k \bar{Q}}^{\mathrm{h}}(t)=-\Delta E_{k \bar{Q}}^{\mathrm{h}} n_{k \bar{Q}}^{\mathrm{h}}(t)+2 V(Q) \Delta f_{k \bar{Q}}^{\mathrm{h}} n(Q, t)
\end{aligned}
$$

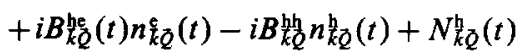

In these equations we have introduced the notation

$$
\begin{aligned}
& \Delta E_{\bar{k}}^{\mathrm{e}(\mathrm{Q})}=\epsilon_{\hat{k}+\not{Q}}^{\mathrm{e}(\mathrm{h})}-\epsilon_{\hat{k}}^{\mathrm{e}(\mathrm{h})}, \\
& \Delta f_{\hat{k}}^{(\mathrm{h})}=f_{\hat{k}+\bar{Q}}^{\mathrm{e}(\mathrm{h})}-f_{\hat{k}}^{\mathrm{e}(\mathrm{h})}, \\
& \epsilon_{k}^{(\mathrm{h})}=h^{2} k^{2} / 2 m_{\mathrm{e}(\mathrm{h})},
\end{aligned}
$$




$$
\begin{gathered}
f_{k}^{\ell}=\operatorname{Tr}\left\{C_{k}^{\ddagger} C_{k} \bar{\rho}\right\}, \\
f_{k}^{k}=\operatorname{Tr}\left\{h_{-k}^{\dagger} h_{k} \bar{\rho}\right\}, \\
V(Q)=4 \pi e^{2} / V \epsilon_{o} Q^{2}, \\
n(\vec{Q}, t)=\sum_{\bar{k}}\left[n_{k \bar{Q}}^{\mathrm{e}}(t)+n_{k \Phi}^{\mathrm{h}}(t)\right],
\end{gathered}
$$

where $\epsilon_{0}$ is the background static dielectric constant, $V$ the volume of the system, and $n(\vec{Q})$ is the amplitude of wave-vector $\vec{Q}$ of the carrier density.

Further, coefficients $B$ contain the effect of laser light absorption, recombination and carrier-phonon interaction; we neglect the latter since, at low temperatures, it produces relaxation effects much smaller than those associated with recombination [5]. Then, coefficients $B$ are $B^{\text {eh }}=B^{\text {hh }} \equiv B^{\mathrm{e}}$ and $B^{\text {he }}=B^{\text {ee }} \equiv B^{\text {h }}$, where

$$
\begin{aligned}
B_{\vec{k}}^{\mathrm{e}(\grave{Q})}= & A_{\mathrm{L}} \delta\left(\epsilon_{k}^{\times}+E_{\mathrm{G}}-\hbar \omega_{\mathrm{L}}\right)+A_{\mathrm{R}}\left(\epsilon_{\vec{k}}^{x}+E_{\mathrm{G}}\right) f_{\hat{k}}^{\text {e(h) }} \\
& + \text { same term with } \vec{k} \leftrightarrow \vec{k}+\vec{Q},
\end{aligned}
$$

and

$$
\begin{aligned}
\epsilon_{k}^{x} & =\hbar^{2} k^{2} / 2 m_{x} \\
A_{\mathrm{L}} & =\left(2 \pi^{2} e^{2} E_{\mathrm{G}} / \epsilon_{\infty} c \omega_{\mathrm{L}}^{2} m_{x}\right) I_{\mathrm{L}} \\
A_{\mathrm{R}} & =e^{2} E_{\mathrm{G}} / \epsilon_{\infty} \hbar c^{3} m_{x}
\end{aligned}
$$

and we recall that we have used the dipolar approximation to deal with the radiation fields.

Finally, in eqns (7) $N^{\text {e(h) }}$ are nonlinear terms given by

$$
\begin{aligned}
& N_{k \bar{Q}}^{e_{Q}}(t)=2 V(Q) \sum_{\bar{Q} \neq \bar{Q}}\left[n_{\bar{k}}^{\mathrm{e}}+\bar{Q}^{\prime}, \grave{Q}-\bar{Q}^{\prime}(t)-n_{k . \bar{Q}-\bar{Q}}^{\mathrm{e}},(t)\right] \\
& \times \sum_{k^{\prime}}\left[n_{k^{\prime}, \emptyset^{\prime}}^{\mathrm{e}}(t)+n_{-k^{\prime},-\bar{Q}}^{\mathrm{h}}(t)\right] \\
& +i \sum_{\bar{Q}^{\prime} \neq \bar{Q}}\left\{A_{\mathrm{L}} \delta\left(\epsilon_{k}^{x}+\bar{Q}^{\prime}+E_{\mathrm{G}}-\hbar \omega_{\mathrm{L}}\right)\right. \\
& \left.+\sum_{\vec{q}} A_{\mathrm{R}} \delta\left(\epsilon_{\bar{k}+\bar{Q}^{\prime}}+E_{\mathrm{G}}-\hbar \omega_{q}\right)\right\} \\
& \times\left[n_{\hat{k}, \bar{Q}^{\prime}}(t) n_{\hat{k}}^{\underline{h}}-\bar{Q}^{\prime} \cdot \bar{Q}^{\prime}-\bar{Q}^{(t)}\right. \\
& \left.+n_{k+Q^{\prime} \cdot \bar{Q}-\bar{Q}^{\prime}}(t) n_{k,-\bar{Q}^{\prime}}^{\underline{h}}(t)\right]
\end{aligned}
$$

$$
\begin{aligned}
& N_{k \emptyset}^{\mathrm{h}}(t)=-2 V(Q) \sum_{Q^{\prime} \neq Q}\left[n_{-k-Q^{\prime}, \bar{Q}^{\prime}-Q}^{\mathrm{h}}(t)\right. \\
& \left.-n_{-k, Q^{\prime}-Q^{\mathrm{h}}}^{(t)}\right] \\
& \times \sum_{\bar{k}^{\prime}}\left[n_{-\bar{k}^{\prime}, \bar{Q}^{\prime}}^{\mathrm{h}}(t)+n_{k^{\prime}, \bar{Q}^{\prime}}^{\mathrm{e}}(t)\right] \\
& -i \sum_{Q^{\prime} \neq \bar{Q}}\left\{A_{\mathrm{L}} \delta\left(\epsilon \frac{\bar{x}}{k}+\bar{Q}^{\prime}+E_{\mathrm{G}}-\hbar \omega_{\mathrm{L}}\right)\right. \\
& \left.+\sum_{\bar{q}} A_{\mathrm{R}} \delta\left(\epsilon_{k+\bar{Q}}+E_{\mathrm{G}}-\hbar \omega_{q}\right)\right\} \\
& \times\left[n_{\hat{k}, Q^{\prime}}^{\mathrm{c}}(t) n_{-k-\bar{Q}^{\prime}, \dot{Q}^{\prime}-\grave{Q}^{\mathrm{h}}}(t)\right.
\end{aligned}
$$

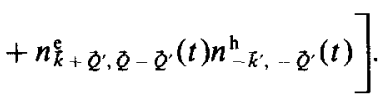

Clearly, the stationary $\left(\partial n^{\text {e(h) }} / \partial t=0\right)$ uniform solution is $n_{\mathrm{k}}^{\text {e(h) }}=0$, a fixed point solution for the dynamic system of carriers. To establish its stability we need to perform linear stability analysis [11]. In particular, since we are looking for a possible branching point (bifurcation) of solutions giving rise to the emergence of a stationary nonuniform state $(n+k)$, we look for the existence of a zero solution in the eigenvalue spectrum of the linearized equations of motion. The latter, because the reference solution is the homogeneous one with $n_{k Q}^{e(b)}=0$, is the coupled set of eqns (7) with putting $N^{\text {e(h) }}=0$. Putting both equations equal to zero (null eigen-

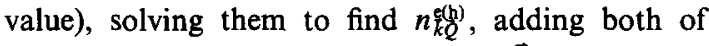
these quantities and summing over $\vec{k}$ we find the characteristic equation

$$
n(\vec{Q}) \epsilon(\vec{Q})=0
$$

where $n(\vec{Q})$ is given by eqn $(8 \mathrm{~g})$ and

$$
\epsilon(\vec{Q})=1-V(Q) \sum_{\vec{k}} M(\vec{k}, \vec{Q}) D^{-1}(\vec{k}, \vec{Q})
$$

where

$$
\begin{aligned}
& M(\vec{k}, \vec{Q})=\Delta f_{k \bar{Q}}^{e_{\bar{Q}}} \Delta E_{k \grave{Q}}^{\mathrm{h}}+\Delta f_{k \bar{Q}}^{\mathrm{h}} \Delta E_{k \bar{Q}}^{e}
\end{aligned}
$$

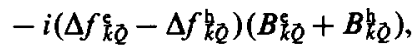

$D(\vec{k}, \vec{Q})=\left(\Delta E_{\vec{k} \bar{Q}}^{\mathrm{e}}-i B_{\vec{k} \underline{\mathrm{h}}}^{\mathrm{h}}\right)$

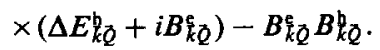


Hence, one solution of eqn (12) is $n(\vec{Q})=0$, i.e. the one corresponding to the uniform state, and $n(\vec{Q})$ can be different from zero, a nonuniform solution, if $\epsilon(\vec{Q})=0$. It is a quite interesting fact to note that $\epsilon(\vec{Q})$ is the static but wave-vector-dependent electronic contribution to the dielectric function: in fact if we introduce a probe charge $-e r_{0} \exp \{-i \vec{Q} \cdot \vec{r}\}$; we add its effect to the cvolution equations, eqns (7), in the stationary regime, namely terms of the form $V(Q) \Delta_{k Q}^{e(b)} r_{0}$; solve the coupled equations to obtain $n(\vec{Q})$, and use Maxwell and constitutive equations one finds that

$$
\epsilon^{-1}(\vec{Q})-1=n(\vec{Q}) / r_{0},
$$

where $\epsilon(\vec{Q})$ is precisely the same as in eq (13). It should be noted that putting $B^{\mathrm{e}(b)}=0$ in eqns (14), the corresponding $\epsilon(\vec{Q})$ becomes the Lindhart (RPA) dielectric function of a system of free electrons and holes; coefficients $B^{\text {e(h) }}$ are the ones that introduce dissipative effects in the dielectric function in place of Landau damping in Lindhart expression, coupling both type of carriers.

The imaginary part of $\epsilon(\vec{Q})$ is null, as it should be, since it is an odd function of $\omega$ [15]. Performing the integrations required by eq (13) we find

$$
\begin{aligned}
\operatorname{Re} \epsilon(\vec{Q})=1-\frac{2 e^{2}}{\pi \epsilon_{0} Q^{2}}\left\{2^{1 / 2}\left(\frac{m_{x}}{\hbar^{2}}\right)^{3 / 2}\right. & I_{1}(Q) \\
& \left.+\frac{m_{x}}{\hbar^{4} Q} I_{2}(Q)\right\}
\end{aligned}
$$

where,

$$
\begin{aligned}
I_{1}(Q)= & \int_{0}^{\infty} \mathrm{d} E E^{1 / 2}\left[f_{\mathrm{e}}^{\prime}(E)-f_{\mathrm{h}}^{\prime}(E)\right] \\
& \times\left[1-C^{-1}(E, Q) \operatorname{arctg} C(E, Q)\right], \\
I_{2}(Q)= & \int_{0}^{\infty} \mathrm{d} E\left[B^{\mathrm{e}}(E)+B^{\mathrm{h}}(E)\right] \\
& \times\left[m_{\mathrm{e}} f_{\mathrm{h}}^{\prime}(E)-m_{\mathrm{h}} f_{\mathrm{e}}^{\prime}(E)\right] \operatorname{arctg} C(E, Q)
\end{aligned}
$$

with

$$
\begin{gathered}
B^{\mathrm{e}(\mathrm{h})}(E)=A_{\mathrm{L}} \delta\left(E+E_{\mathrm{G}}-\hbar \omega_{\mathrm{L}}\right) \\
+A_{\mathrm{R}}\left(E+E_{\mathrm{G}}\right) f_{\mathrm{e}(\mathrm{h})}(E), \\
C(E, Q)=\hbar Q \sqrt{2 m_{x} E} /\left[m_{\mathrm{e}} B^{\mathrm{h}}(E)-m_{\mathrm{h}} B^{\mathrm{e}}(E)\right],
\end{gathered}
$$

$$
\begin{gathered}
f_{\mathrm{e}(\mathrm{h})}^{\prime}(E)=\partial f_{\mathrm{e(h)}}(E) / \partial\left(\frac{m_{x}}{m_{\mathrm{e}(\mathrm{h})}} E\right) \\
f_{\mathrm{e(h)}}(E)=\left[1+\exp \beta\left(\frac{m_{x}}{m_{\mathrm{e}(\mathrm{h})}} E-\mu_{\mathrm{e}(\mathrm{h})}\right)\right]^{-1} .
\end{gathered}
$$

In the classical limit and with $Q$ going to zero, we find that eqn (16) becomes

$$
\operatorname{Re} \epsilon(\vec{Q}) \simeq 1+\frac{8 \pi n e^{2}}{\epsilon_{0} m_{x} Q^{2}} \equiv 1+\frac{Q_{\mathrm{DH}}^{2}}{Q^{2}},
$$

where $Q_{\mathrm{DH}}$ is the Debyc-Hückel screening wavenumber, and so we recover the well-known result for the free (double component) electron gas, and no instability can occur.

In general, in the limit of small $Q$, and using in eqns $(17 \mathrm{a}, \mathrm{b})$ arctg $x \simeq x-x^{3} / 3$, we obtain the approximate expression

$$
\operatorname{Re} \epsilon(Q) \simeq 1+A+\frac{\alpha}{Q^{2}} \equiv \epsilon_{\mathrm{p}}\left[1+\frac{Q_{0}}{Q^{2}}\right],
$$

where

$$
\begin{gathered}
A=\frac{2^{5 / 2} e^{2} m_{x}^{5 / 2}}{\pi 3 \epsilon_{0} \hbar} \int_{0}^{\infty} \mathrm{d} E E^{3 / 2}\left[\frac{\left|f_{\mathrm{e}}^{\prime}(E)+f_{\mathrm{h}}^{\prime}(E)\right|}{\left[m_{\mathrm{e}} B^{\mathrm{e}}(E)-m_{\mathrm{h}} B^{\mathrm{h}}(E)\right]^{2}}\right. \\
\left.-\frac{\left[B^{\mathrm{e}}(E)+B^{\mathrm{h}}(E)\right]\left|m_{\mathrm{e}} f_{\mathrm{h}}^{\prime}(E)-m_{\mathrm{h}} f_{\mathrm{e}}^{\prime}(E)\right|}{\left[m_{\mathrm{e}} B^{\mathrm{h}}(E)-m_{\mathrm{h}} B^{\mathrm{e}}(E)\right]^{3}}\right] \\
\alpha=\frac{2^{3 / 2} e^{2} m_{\mathrm{x}}^{3 / 2}}{\pi \epsilon_{0} \hbar^{3}} \int_{0}^{\infty} \mathrm{d} E \mid m_{\mathrm{e}} f_{\mathrm{h}}^{\prime}(E) \\
-m_{\mathrm{b}} f_{\mathrm{e}}^{\prime}(E) \mid \frac{E^{1 / 2}\left[B^{\mathrm{e}}(E)+B^{\mathrm{h}}(E)\right]}{m_{\mathrm{e}} B^{\mathrm{e}}(E)-m_{\mathrm{h}} B^{\mathrm{h}}(E)}, \quad \\
\epsilon_{\mathrm{p}}=1+A ; \quad Q_{0}^{2}=\alpha / \epsilon_{\mathrm{p}}
\end{gathered}
$$

Therefore, $\epsilon(\vec{Q})$ is always positive and consequently the stationary homogeneous state is stable against the formation of spatial ordering.

But, from another point of view, we can note that the dielectric function tends to be smaller than the purely metallic one due to Lindhart for the free double plasma. Furthermore, let us consider the extremely degenerate limit (very low temperatures and high densities); then $\alpha$ in eqn (20b) becomes very small and $\epsilon(\vec{Q})$ is much smaller than the values it would have in the case of the free (double component) electron gas, the latter being $1+\left(Q_{\mathrm{FT}} / Q\right)^{2}$, where $Q_{\mathrm{FT}}$ is the Fermi-Thomas reciprocal screening length. 
In the limit of $Q$ going to zero, and using Fermi-Dirac step functions for the carrier distribution functions in eqn (16) we obtain

$$
\begin{aligned}
\operatorname{Re} \epsilon(\vec{Q})= & +\frac{2 e^{2}}{3 \pi \epsilon_{0} Q^{2}} \frac{m_{x}^{3 / 2}}{\hbar^{3}} \int_{0}^{\infty} \mathrm{d} e E^{1 / 2} \\
& \times\left[\delta\left(\frac{m_{x}}{m_{\mathrm{e}}} E-\epsilon_{\mathrm{F}}^{\mathrm{e}}\right)+\delta\left(\frac{m_{x}}{m_{\mathrm{h}}} E-\epsilon_{\mathrm{f}}^{\mathrm{h}}\right)\right] \\
& \times C^{2}(E, Q)+\frac{2 e^{2} m_{x}}{\pi \epsilon_{0} \hbar^{4} Q^{3}} \int_{0}^{\infty} \mathrm{d} E\left[m_{\mathrm{e}}\right. \\
& \left.\times \delta\left(\frac{m_{x}}{m_{\mathrm{h}}} E-\epsilon_{\mathrm{F}}^{\mathrm{h}}\right)-m_{\mathrm{h}} \delta\left(\frac{m_{x}}{m_{\mathrm{e}}} E-\epsilon_{\mathrm{F}}^{\mathrm{e}}\right)\right] \\
& \times\left[B^{\mathrm{e}}(E)+B^{\mathrm{h}}(E)\right] C(E, Q)
\end{aligned}
$$

where $\epsilon_{\mathbf{F}}^{\mathrm{e}(\mathrm{l})}$ are the electron (hole) Fermi energies.

But in the last term the integral is null, because it is null the first square bracket in its integrand, the other integral is independent of $Q$ (let us recall that $C \sim Q$ ), and therefore $\epsilon(Q)$ is a constant for $Q$ going to zero. The infinity of $\epsilon(\vec{Q})$ for infinitely long wavelengths in eqn (19) is a manifestation of the metallic character of the system. On the contrary, its finite value in eqn (21) tells us that the system has dielectric (non-metallic) properties.

The resulting nonmetallic carrier system should resemble an excitonic-like nonequilibrium phase with electrons and holes bounded in a nonconducting polarizable state. In the intermediate degenerate regime the screening factor has finite nonvanishing values; the static dielectric constant is again infinite at $Q=0$, but $\epsilon(\vec{Q})$ remains below the values of the one

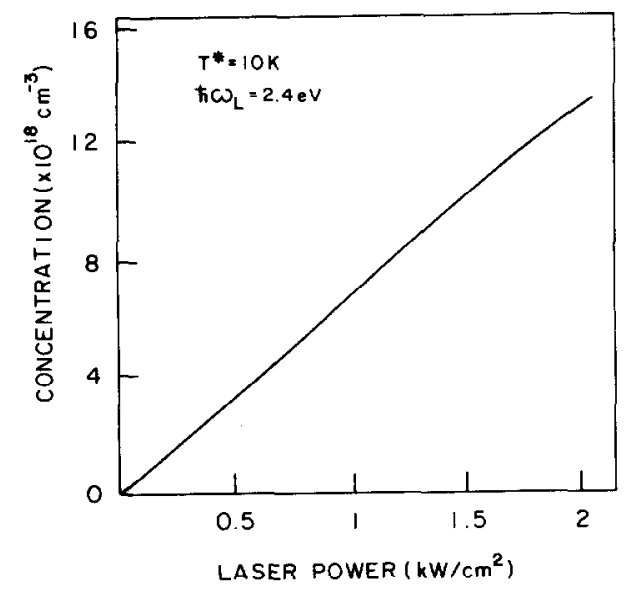

Fig. 1. The concentration of photoinjected carriers as a function of the laser power in GaAs; carrier temperature is $10 \mathrm{~K}$.

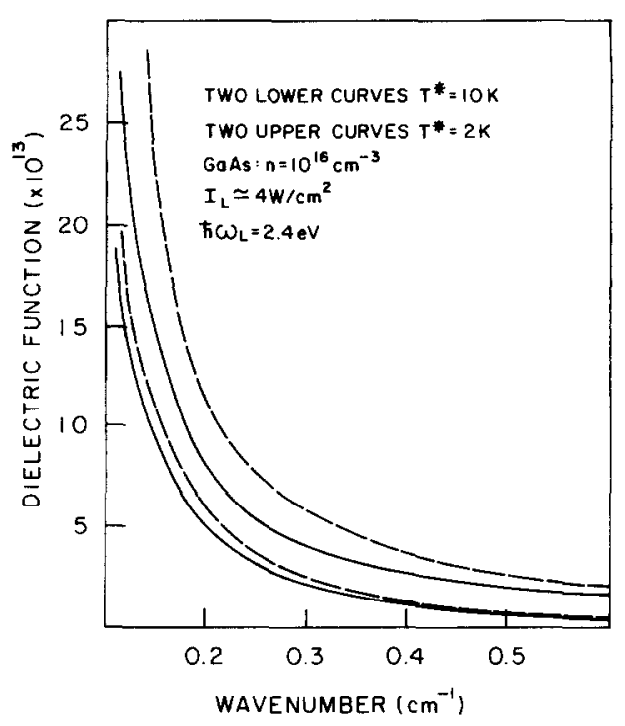

Fig. 2. Dependence of the real part of the static dielectric function on the wavevector, for $T^{*}=2 \mathrm{~K}$ (lower curves) and $T^{*}=10 \mathrm{~K}$ (upper curves), and $n=10^{16} \mathrm{~cm}^{-3}$. For each pair of curves the dashed one corresponds to the Lindhart value for the free double fluid of carriers.

corresponding to the ideal double gas of free carriers. Thus it is conjecturable that finite temperature breaks the bound state of electron-hole pairs leading to the formation of a nonequilibrium phase with coexistence of nonmetallic bounded electrons and holes and metallic itinerant carriers.

We perform next numerical calculations, using parameters appropriate to $\mathrm{GaAs}$, illuminated by a laser of photon frequency $\hbar \omega_{\mathrm{L}}=2.4 \mathrm{eV}$. In Fig. 1, the dependence of carrier photoconcentration on the laser power $I_{\mathrm{L}}$ is shown, obtained using eqn (1) and $T=10 \mathrm{~K}$. The first part of the curve (low densities) displays the linear dependence of $n$ with $I_{\mathrm{L}}$ expected in the semiclassical limit. With increasing laser power, and therefore resulting increasing concentrations, the carrier system is becoming a degenerate quantum gas, and optical saturation follows for $n$ nearly $2 \times 10^{19} \mathrm{~cm}^{-3}$ and $I_{\mathrm{L}} \sim 20 \mathrm{~kW} \mathrm{~cm}{ }^{-2}$.

In Fig. 2 we show the $Q$-dependent dielectric function of eqn (19) (full line) for carrier quasitemperatures $T^{*}$ of $2 \mathrm{~K}$ and $10 \mathrm{~K}$. For comparison we have drawn the Lindhart dielectric function for the double free carrier system, i.e. $B=0$ in eqn (19) (dashed line). We have used $n=10^{16} \mathrm{~cm}^{-3}$, which can be obtained by continuous illumination by laser light of photon energy $2.4 \mathrm{eV}$ and power intensity of, roughly $4 \mathrm{~W} \mathrm{~cm}^{-2}$ [from Fig. 1]. Inspection of Fig. 2 tells us that with increasing carrier quasitemperature $\epsilon(\dot{Q})$ tends to coincide with Lindhart's $\epsilon_{\mathrm{L}}(\vec{Q})$. This reinforces our previous argument that between the nonmetallic state at the extrcmely degenerate 
regime and the classical regime the carrier system is in a nonequilibrium thermodynamic phase with coexistence of carriers in both metallic and nonmetallic states. Using a simple model of two independent fluids for both nonequilibrium phases, we can obtain an estimative of the fraction of carriers in the condensate, i.e. in the nonequilibrium phase of bounded elcetrons and holcs, say $\Delta(\beta, n)=n^{*} / n$ : putting $\epsilon(\vec{Q})=\Delta \epsilon_{\text {cond }}(\vec{Q})+(1-\Delta) \epsilon_{\mathrm{L}}(\vec{Q})$, and considering with $Q$ going to the zero limit, that $\epsilon_{\text {cond }} \ll \epsilon_{\mathrm{L}}$ (since the latter go to infinite and the former is finite), we find that

$$
\Delta(\beta, n)=\lim _{Q \rightarrow 0} \frac{\epsilon_{\mathrm{L}}(\vec{Q})-\epsilon(\vec{Q})}{\epsilon_{\mathrm{L}}(\vec{Q})}
$$

This parameter is displayed in Fig. 3 (obtained for $Q=1 \mathrm{~cm}^{-1}$ ) as a function of the carrier quasitemperature for $n=10^{16} \mathrm{~cm}^{-3}$. For small values of $T^{*}$ the resulting curve can be approximated by

$$
\Delta(\beta, n) \simeq \exp \left[-T^{*} / T_{\mathrm{c}}(n)\right]
$$

where $T_{\mathrm{c}}(n)$ is a characteristic temperature, dependent on $n$, in this case given by $T_{\mathrm{c}}\left(10^{16} \mathrm{~cm}^{-3}\right) \simeq 3.6 \mathrm{~K}$. Thus, for values of $T^{*}$ of the order and larger than this $T_{c}$ the system is in the almost completely metallic state. Furthermore, it should be noticed that if the effective masses $m_{\mathrm{e}}$ and $m_{\mathrm{h}}$ are equal, $C^{-1}$ in eqns (21) is null, $I_{2}$ is also null, and the dielectric function simply reduces to the value

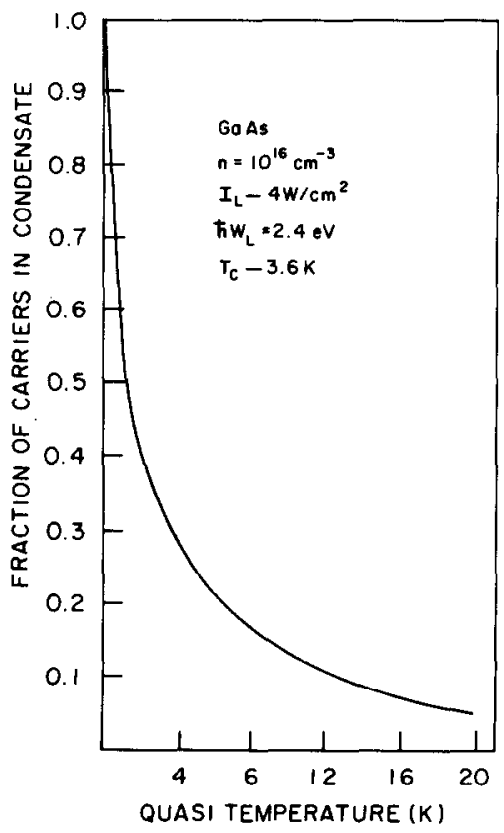

Fig. 3. The fraction of carriers in the polarizable nonconducting nonequilibrium phase as obtained from eqn (23). that corresponds to the one of a metallic carrier gas with concentration $2 n$, and no condensation occurs. Thus, the symmetric condition $m_{\mathrm{e}}=m_{\mathrm{h}}$ cancels the effect of coupling of electron and holes mediated by recombination effects, i.e. coming from the terms containing coefficients $B$.

\section{CONCLUDING REMARKS}

As shown, the photoinjected carriers in a plasma in a semiconductor form a nonequilibrium (dissipative) open system governed by nonlinear dynamic laws for a certain set of basic variables, deemed appropriate for a description of the evolution of the macroscopic state of such a system. Thus, it may be capable of displaying complex behavior in the sense discussed in $[1,2,4]$. We have concentrated our attention on the study of the stationary and uniform carrier density that should be formed on the metallic side of the Mott transition in semiconductors illuminated by continuous laser light, producing electron-hole pairs in photon absorption processes, i.e. a double fluid of itinerant carriers.

We have characterized the nonequilibrium thermodynamic state of the carrier system by the energy, concentration, and the nondiagonal elements of the one quasi-particle density matrix. The latter permits the calculation of the space dependence, in a nonuniform situation, of the dynamical variables of the system, for example the local charge density. The equations of evolution for these basic variables were obtained using the nonlinear quantum transport theory that can be derived from the nonequilibrium statistical operator method [6] in Zubarev's approach [9] and the linear theory of relaxation [10]. We have omitted the equation for the energy (see [5]) taking the carrier quasi-temperature as a parameter, and expecting that after a certain transient it would attain a value near that of the reservoir [5]. The equation that determines the stationary value of the photoconcentration is eqn (1), and eqns (7) that of the one quasiparticle density matrix displaying a particular nonlinearity of a polynomial type, i.e. bilinear terms in the Fourier amplitudes of the charge density resulting from polarization effects associated with the Coulomb interaction, and dissipative effects associated with the interaction of the carriers with the laser and recombination radiation fields.

Resorting to linear normal mode analysis [11] we have shown that the stationary uniform state is stable against the formation of any possible patterned spatial stationary structure. But in the process, through the examination of the static but wave-vector dependent dielectric function that appears in the characteristic equation in our normal mode analysis, 
we are able to show that in the extremely degenerate regime the uniform steady state itinerant Mott metallic phase of carriers suffers a nonequilibrium phase transition to a nonmetallic state. It resembles the formation of a polarizable nonmetallic state, with electrons and holes bounded in an extended structure on a macroscopic scale. Noticing that we have used plane waves for the electrons and hole states, it is conjectured that in this extremely degenerate regime the condensation in the nonmetallic nonequilibrium phase corresponds to the formation of two interpenetrated infinite-wavelength lattices clamped together. This suggests that, in the other extreme of a tight binding approximation for the hole system, the condensed dissipative structure would resemble a gas of Frenkel excitons.

As discussed in the last section, the behavior of the dielectric function on leaving the extremely degenerate regime points to the presence of two coexisting nonequilibrium phases: with increasing temperature electrons and holes are freed from the nonmetallic condensate and the state is partially metallic. Such a fraction of electrons and holes are individually itinerant in the background of the condensed dielectric state. For the case of GaAs, and $n=10^{16} \mathrm{~cm}^{-3}$ (for $I_{\mathrm{L}} \sim 4 \mathrm{~W} \mathrm{~cm}^{-2}$ ) we show in Fig. 3 our estimate of the fraction of carriers in the dielectric condensate. Numerical fitting seems to point to a kind of binding energy of roughly $4 \mathrm{~K}$. It should be noted that this is a different phenomenon than the Keldysh transition (electron-hole droplet formation) in Si and Ge [16].

Summarizing: at a given carrier quasi-temperature with increasing laser power, and therefore increasing concentration of photoinjected carriers, as known, Schottky excitons (and exciton complexes) are formed at low to intermediate concentrations. With increasing laser intensity a critical point is approached (when roughly the orbital radius of Schottky excitons overlap each other) corresponding, it is assumed, to the ionization of the excitons and the appearance of a metallic state of the carriers. Our results change this picture, showing that in the extremely degenerate regime the carriers are not freed at the Mott critical point but tend to form a nonmetallic state of Frenkel excitons. The binding energy of these Frenkel excitons seems to be small and, on leaving the extremely degenerate regime, are rapidly broken liberating electrons and holes as free carriers. Near the extremely degenerate regime the coexistence of both nonequilibrium phases is expected, the dielectric condensate and the fraction of itinerant carriers. A short preliminary report of these results has already been published [17].
As a final word, the fundamental role of the dissipative terms [those with coefficients $B$ in eqns (7)] in the formation of the condensate must be stressed. Paraphrasing Prigogine et al., dissipation, contrary to what is unusually thought, is not a source of decay, but, it must be emphasized, may have quite a constructive role.

Acknowledgements-Financial support from the Brazilian Ministry of Education-MEC/CAPES (ASE) and the Brazilian National Research Council C CNPq (ARV,RL) is gratefully acknowledged.

\section{REFERENCES}

1. Nicolis G. and Prigogine I., Exploring Complexity. Freeman, San Francisco (1989); Nicolis G., Physics of far-from-equilibrium systems and selforganization, in The New Physics (Edited by Davies P). Cambridge Univ. Press, Cambridge (1988); Glansdorff P. and Prigogine I., Thermodynamics of Structure, Stability, and Fluctuations. Wiley, New York (1971); Nicolis G., Rep. Prog. Phys. 49, 873 (1986).

2. Haken H., Synergetics. Springer, Berlin (1978).

3. Prigogine I., Introduction to the Thermodynamics of Irreversible Processes. Wiley, New York (1961).

4. Prigogine I., Nature 246, 47 (1973); Int. J. Quantum Chem. Symp. 9, 443 (1975); Nicolis G. and Prigogine I., Selforganization in Nonequilibrium Systems. Wiley, New York (1977).

5. Tomé T., Vasconcellos A. R. and Luzzi R., Physica 144B, 376 (1987).

6. Luzzi R. and Vasconcellos A. R., Fortschr. Phys./Prog. Phys. 38, 887 (1990): in this article we review the NSOM and discuss the possibility of encompassing the different approaches under a unifying variational principle; a quantum nonlinear theory of transport derived from the NSOM is given in Lauck L., Vasconcellos A. R. and Luzzi R., Physica 168A, 789 (1990), while the connection of the NSOM with existing phenomenological irreversible thermodynamic theories is discussed in Vasconcellos A. R., Luzzi R. and Garcia-Colin L. S., Phys. Rev. A 43, 6622, 6633 (1991).

7. Algarte A. C. and Luzzi R., Phys. Rev. B27, 7563 (1983); Luzzi R. and Vasconcellos A. R., in Semiconductors Probed by Ulirafast Laser Spectroscopy I (Edited by Alfano R. R.); Luzzi R., in High Excitation and Short Pulse Phenomena (Edited by Pilkuhn H. M.). North Holland, Amstcrdam (1985).

8. Freire V. N., Vasconcellos A. R. and Luzzi R., Phys. Rev. B39, 13,264 (1989); Nuovo Cim. 12D, 1387 (1990).

9. Zubarev D. N., Neravnovesnaia Statisticheskaia Termodinamika. Izd. Nauka, Moscow (1971) [English Transl.: Nonequilibrium Statistical Thermodynamics. Plenum Press, New York (1974)].

10. Zubarev D. N., in [9], Chap IV, Section 25.1; also Kalashnikov V. P.. Theor. Math. Phys. (URSS) 35, 362 (1978), and second reference of [6].

11. Davies H. T., Nonlinear Integral and Differential Equations. Dover, New York (1962); also last reference of [4].

12. Elci A., Scully M. O., Smirl A. L. and Matter J. C., Phys. Rev. B16, 191 (1977); Collet J., Amand T. and Pugnet M., Phys. Lett. A96, 368 (1983).

13. Algarte A. C., Vasconcellos, and Luzzi R., Proc. 18th Int. Conf. Phys. Semiconductors (Edited by Engström O.) Vol. 2, pp. 1295-1298. World Scientific, Singapore (1986). 
14. Blackmore L. S., Semiconductor Statistics. Pergamon Press, Oxford (1962).

15. Landau L. D. and Lifshitz E. M., Electrodynamics of Continuous Media. Addison-Wesley, Reading, Massachusetts (1960).
16. Keldysh L. V., Proc. Ninth Int. Conf. Phys. Semiconductors (Edited by Ryukin S. M. and Shmastsev V. V.), p. 1303. Izd. Nauka, Leningrad (1968).

17. Esperidião A. S., Vasconcellos A. R. and Luzzi R., Solid State Commun. 73, 673 (1990). 